\title{
Qualitative Analysis of Age and Brand of Unblended Brandy by Electronic Nose
}

\author{
Yang Yang*, Yu Zhao*, Shuming Zhang, Yuanying Ni, and Jicheng Zhan ${ }^{* *}$ \\ College of Food Science and Nutrient Engineering, National Engineering Research Center \\ for Fruit and Vegetable Processing, China Agricultural University, Beijing 100083, China
}

\begin{abstract}
This paper reports the capability of electronic nose based on gas chromatograph (GC-Flash) in age identification and brand classification of brandy. Three different kinds of brandies by the static headspace sampling for the injection of the volatile compounds were analyzed. The data were disposed by multivariate data processing based on principal component analysis (PCA) and cluster analysis (CA). The results show that PCA could identify the age as PC1 represented the raw information of age well; PCA on small difference ages of the samples could classify the brands of the samples. The CA would cluster samples by age through its analysis on the three different kinds of brandies respectively; The CA on all samples indicated that significant differences existed among different brands with age under 20 years and clustering process performed firstly in each group by age information and then clustering progressively by age among all groups. GC-Flash electronic nose can be applied to identify the brand and age of different brandies.
\end{abstract}

Keywords: electronic nose, brandy, principal component analysis, cluster analysis.

\section{Introduction}

Grape brandy is a kind of alcoholic beverage, taking grapes as raw material and whose formation follows fermentation, distillation and storing in oak barrels [1]. The unblended liquor after wine aging is namely original wine, which in the storing undergoes complicated physical, chemical and biological changes. The assessment by chemical methods and sensory evaluation is highly time-, labour- and capitalconsumed; therefore the age of wine is regarded as a significant index to determine the quality and class of brandy [2]. With the development of detecting instruments, the rapid analysis technology (electronic nose, electronic tongue, electronic eyes and near-infrared spectroscopy, etc.), based on rapid analysis of the total sample information ("fingerprint"), is gradually applied to rapid identification of wine age, quality, origin and brand.

Electronic nose comprises an array of electronic sensors with partial specificity and an appropriate pattern-recognition system, capable of recognizing simple or complex

\footnotetext{
Yang Yang and Yu Zhao contributed equally to this work.

Corresponding author. 
odors [2]. Electronic nose technology is mainly applied in the food industry in quality control, process monitoring, freshness evaluation, shelf life prediction, authenticity assessment, etc.[2].Researches in fermentation monitoring[3-7], identification of brand [8-10] and origin[11-13], aroma evaluation [13-16] and false distinguishing[17] by electronic nose have been carried out at home and abroad with excellent performance. Electronic nose, as a rapid qualitative analysis instruments at first, after decades of development, has also made certain progress in quantitative analysis. The mass spectrometry-based electronic nose invented in the nineties [5, 18], stepped forward a great deal in quantitative analysis.

The former researches on wine analysis by electronic nose have focused on the sensor-based or mass spectrometry-based electronic nose, but seldom on the ones analyzing brandy, and even the research on testing original brandy has not been reported yet. This study, using gas chromatography-based electronic nose, carried out studies on the wine age and brand identification of 3 domestic and overseas brands for the basis of wider application of electronic nose.

\section{Experimental}

\subsection{Samples}

The studies were performed on 29 brandies from three different brands (Group A, $\mathrm{n}=12$; Group B, $\mathrm{n}=13$; Group C, $\mathrm{n}=4$ ). Group A, France Brandy, of which the ageing time is from 4 to 46 years old. Group B and Group C are made in China, whose ageing time are 2 to 15 and 1 to 5 , respectively. Those samples (brandies) came from the same variety of grape (Ugni Blanc) and the same traditional brewing process applied in Cognac region in France. They are all raw brandies without blending after maturing in oak barrels. The samples were taken directly from the winery and then stored at $4^{\circ} \mathrm{C}$. All samples were kept at $20^{\circ} \mathrm{Cfor} 2 \mathrm{~h}$ before testing.

\subsection{Electronic Nose}

Experiments were performed with the HERACLES Flash Electronic nose (Alpha M.O.S Company, France) consisted of a Heracles host machine and a headspace auto-sampler unit. The HERACLES is a highly selective and sensitive specialty gas chromatograph, capable of performing very fast, low level hydrocarbon measurements in laboratory or field environments. Key features include versatility and speed of analysis, giving the productivity advantages over the more traditional gas chromatographs. The HERACLES is a programmed temperature gas chromatograph using syringe or valve inlets to a flash evaporator. The sample is delivered to an adsorbent trap to concentrate the sample for delivery to twin capillary columns and flame ionization detectors (FID) simultaneously [19]. Trace level gas phase hydrocarbon samples down to a few parts per thousand million can currently be measured. Instrument control and the raw data process were performed by Alpha Soft (V11, Alpha M.O.S). A general scheme of the HERACLES Flash Electronic nose is presented in Fig. 1. 


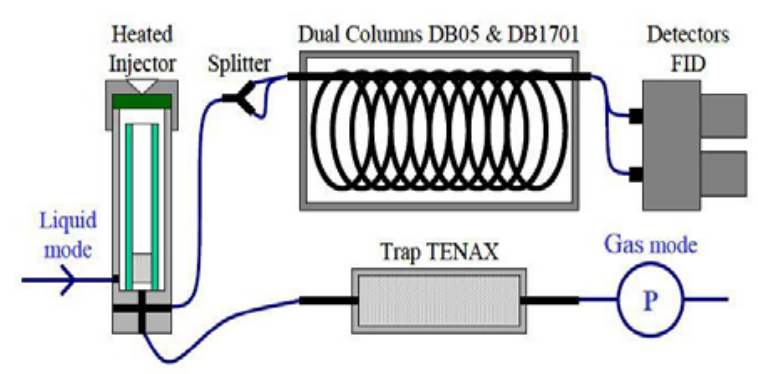

Fig. 1. Set-up applied in the measurement

In order to obtain maximum sensor response, operating parameters for the electronic nose were optimized for the brandy samples. The analysis parameters are as follows: (1) Injector: injection time $1500 \mathrm{~ms}$, injector temperature $200{ }^{\circ} \mathrm{C}$, injection volume $2000 \mu \mathrm{L}$; (2) Temperature program: initial temperature $40{ }^{\circ} \mathrm{C}$, final temperature $200{ }^{\circ} \mathrm{C}$, heating rate $2.0^{\circ} \mathrm{C} / \mathrm{s}$; (3) Detector temperature: $220^{\circ} \mathrm{C}$; (4) Trap temperature: $250{ }^{\circ} \mathrm{C}$, purge time $15 \mathrm{~s}$. (5) Acquisition time: $84 \mathrm{~s}$.

For the measurements, $1000 \mu \mathrm{L}$ of the sample was placed in a $10 \mathrm{~mL}$ glass vial provided with a pierceable disk in the cap. Before gas sample injection, sample was agitated by the auto-sample unit at $40( \pm 0.2){ }^{\circ} \mathrm{C}, 500 \mathrm{rpm}$. All samples were analyzed in 7 times and the average of the chromatograms was used for subsequent statistical analysis.

\subsection{Data Analysis}

Two pattern recognition methods, principal component analysis (PCA) and Cluster analysis (CA) have been used for data analysis. Principal component analysis (PCA) is a chemometric linear, unsupervised and pattern recognition technique used for analyzing and reducing the dimensionality of numerical datasets in a multivariate problem. It can observe the classification results of sample principal components through the PCA map. PCA was performed in Alpha Soft (V11, Alpha M.O.S, France). Cluster analysis (CA) is a kind of unsupervised pattern recognition. It is a method for grouping objects of similar kind into respective categories. CA of Samples was performed using SAS 9.2 software.

\section{Results and Discussion}

Detect and analyze Group A, B, C by electronic nose, eliminate abnormal data [20], obtain the peak figure (retention time and peak area) for each sample. After deducting the blank peak 24 peaks were obtained, including 12 peaks from gas spectrum column 1 and 12 peaks from column 2. Retention time and peak area of those 24 peaks are the "fingerprint" of static headspace volatile components which acquired by electronic nose, and also the raw data of principal component analysis and clustering analysis. For better understanding, the 24 retention time obtained by electronic nose can be defined as sensors while the corresponding peak area can be defined as the response value of sensors. 


\subsection{Principal Component Analysis Results}

In this study, the PCA was firstly applied to group A. Using the raw data directly, three principal components were extracted: PC1-68.36\%, PC2-9.90\%, PC3-7.59\%, The ellipses, which correspond to the $85.85 \%$ confidence intervals, are basically reflect the raw information [21]. Fig. 2 demonstrates the score plot of the first two principal components of Group A. It is easy to distinguish brandy by their different ageing time and furthermore, with the extension of ageing time, the trends appear obviously in PC1: on the PC1 axis from right to left. Meanwhile, samples of close age which are distributed concentrated in the Fig. 2 and can be divided into 4 groups. Information from PC2 is inefficient in distinguishing samples of 30,36 and 39 years and the supplement from PC2 turns to be necessary.

The PCA analysis results displays the similar scene in Fig. 3, four principal components were extracted: PC1-45.32\%, PC2-18.34\%, PC3-15.19\%, PC4-6.29\%, and $85.04 \%$ of the total variance of the data is displayed in the plot. The PCA analysis on Group A and B show that electronic nose is able to distinguish the ageing time of two brandies respectively clearly and the collected first principle components represent ageing time well. The PCA analysis on Group C represent an even better results, however, because of the limited volume of samples, the results are not listed in this paper.

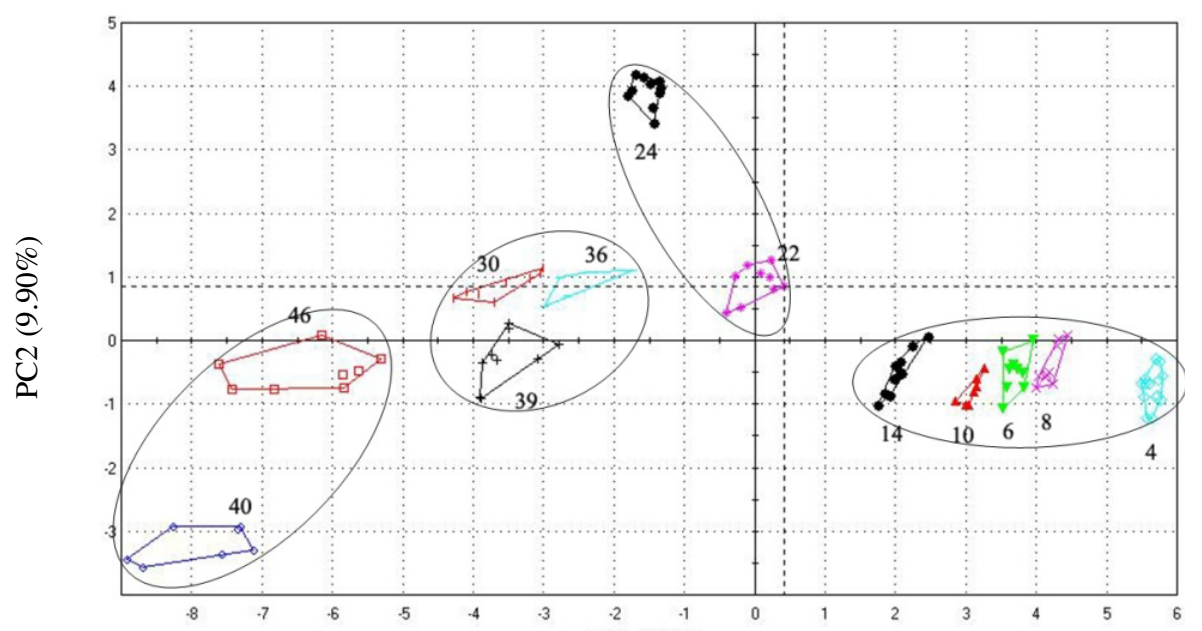

PC1 $(68.36 \%)$

Fig. 2. Score plot of the first two principal components of Group A. The number $4,6,8, \ldots, 46$ represents for the Brandies' ageing time. 


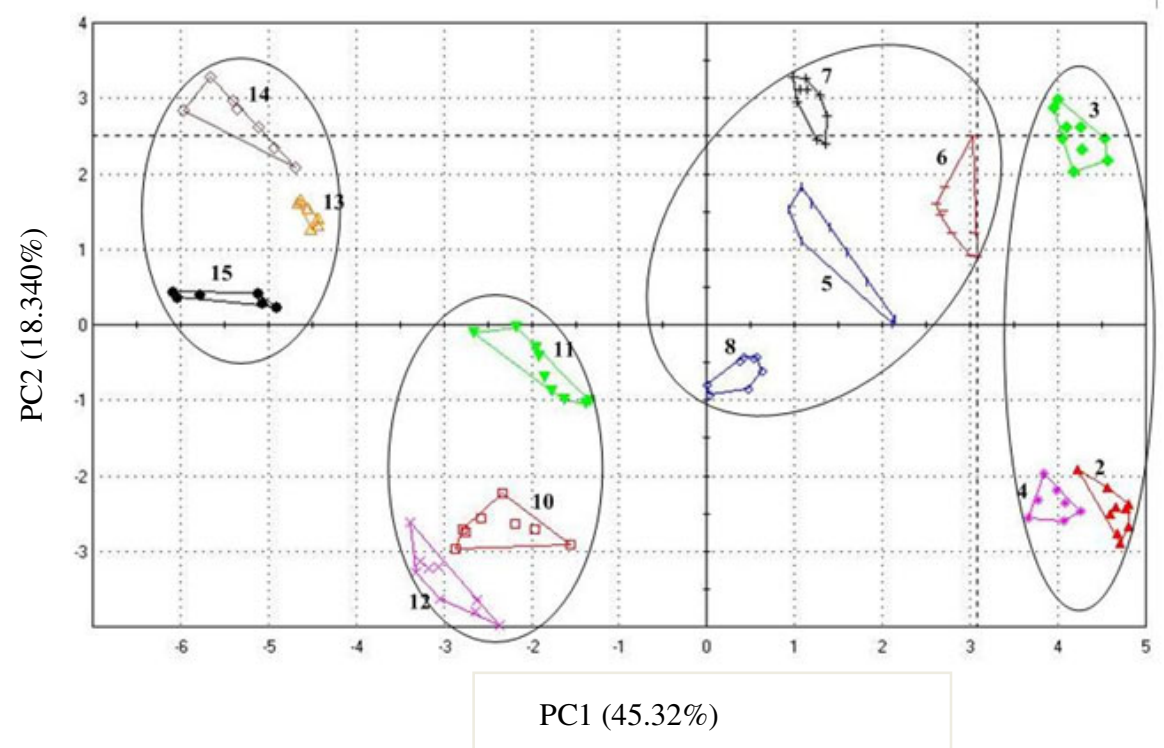

Fig. 3. Score plot of the first two principal components of Group B. The number 2, 3, 4, ,.,15 represents for the Brandies' ageing time.

In the analysis process, we optimized sensors (eliminate the small peak with poor reproducibility and strongly linear-correlated sensor) and screened 12 sensors out of 24 sensors. Finally extracted two principal components: PC1 -70.32\%, PC2-19.20\% and the cumulative contribution rate is $89.52 \%$. Fig. 4 shows the score and loading map of PCA analysis, in which we can see that electronic nose can distinguish the age and brand of different design groups. PC1 axis indicates information of wine ages and PC2 axis indicates information of brands. But PC2 is insufficient to differentiate the aged wine in Group B and the wine in Group A, which, is possibly relevant to the difference of wine ages among samples. At the same time, the loading map shows the information of ageing time and brand of sensors (retention time and peak area of electronic nose). In order to study the brand-recognition capacity of electronic nose for original brandies, we designed analysis process including samples of the three brands. Due to the large difference among the 3 groups of wine, Group A (aged for 4 and 6 years) and Group B (aged for 2,3,4,5,6 years) and Group C(aged for 1,2,3,5 years) were chosen to conduct PCA . The peak value at $4.17 \mathrm{~s}, 10.35 \mathrm{~s}, 14.17 \mathrm{~s}, 16.62 \mathrm{~s}, 21.79 \mathrm{~s}$ of Column gas spectrum 1 and peak value at $4.53 \mathrm{~s}, 8.30 \mathrm{~s}$ of Column gas spectrum 2 represent more brand information while the left 5 peak value represent ageing time information.

Fig. 5 is the score plot of PCA after decreasing the samples difference in wine age. The afterwards results illustrated a better effect in distinguishing, which means that ageing time difference will effect analysis results in brands distinguishing, overlarge difference in sample ages brings a great deal of difficulties to distinguishing brand information among different groups by electronic nose. 


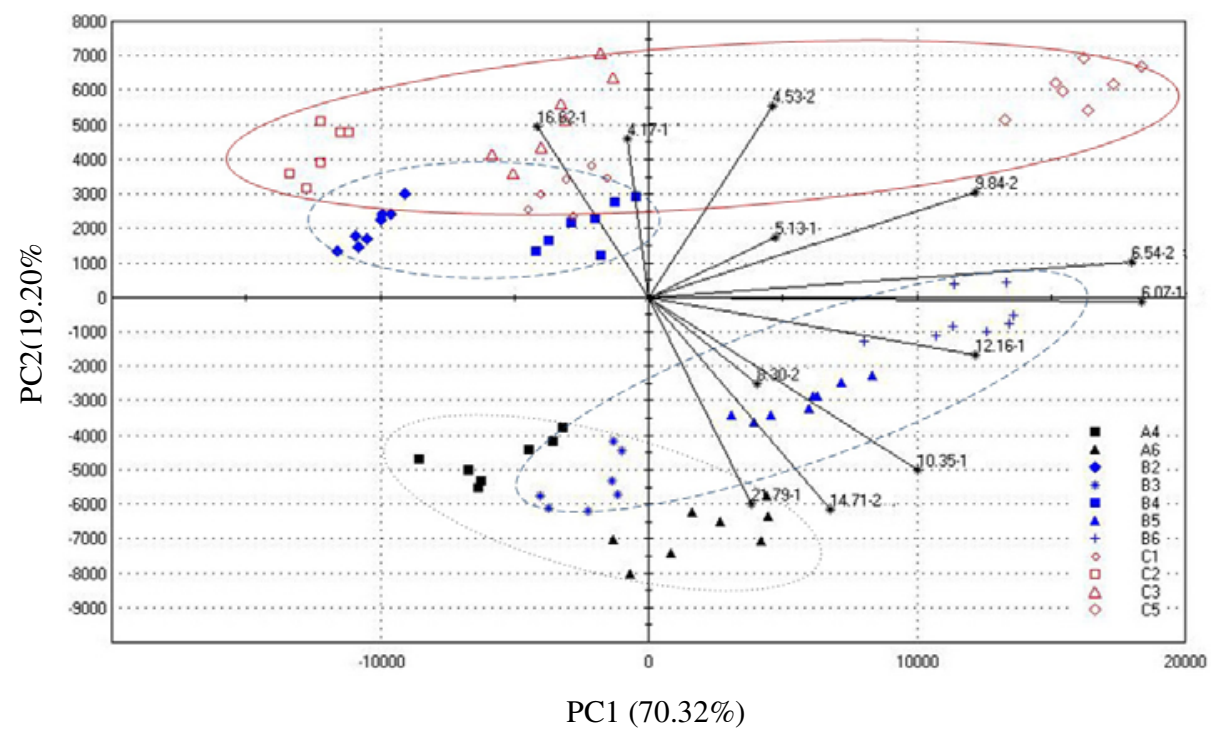

Fig. 4. Score and loading plots of PCA for Group A, B and C. The figure represents for the retention time of peak and the number of column. (e.g. 4.35-2 represents the peak value at $4.53 \mathrm{~s}$ of Column gas spectrum 2).

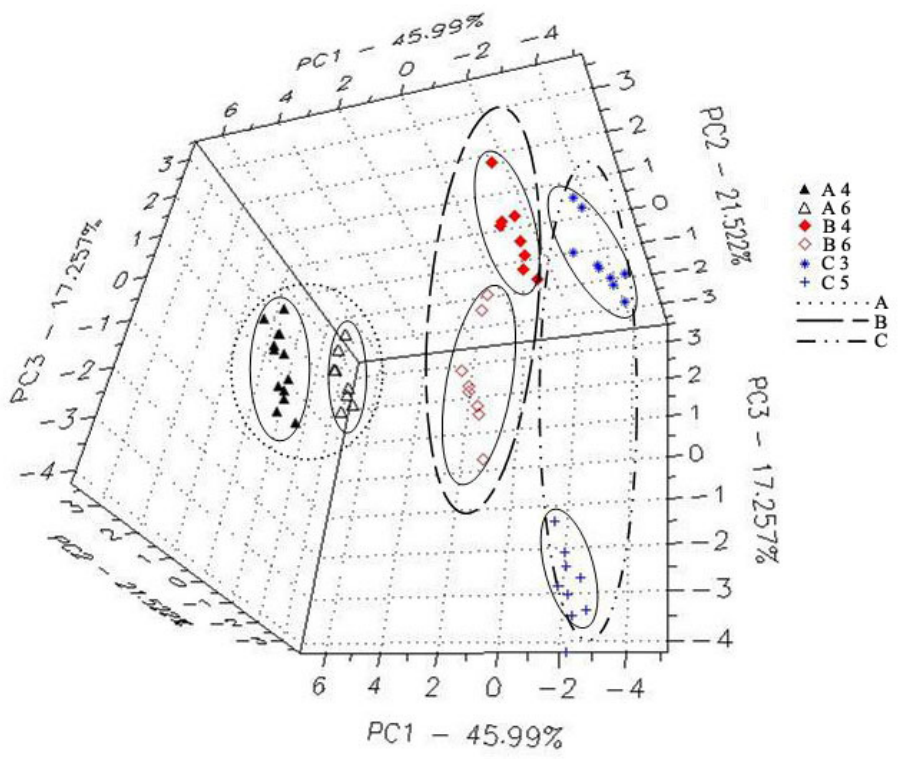

Fig. 5. Score plots of PCA for Group A, B and C 


\subsection{Clustering Analysis Result}

In order to excavate more test results of the original brandy from the electronic nose, cluster analysis method was applied in Group A and equally in Group A, B and C respectively. Cluster analysis belongs to unsupervised pattern recognition, through which man can observe the effects of automatic classification and recognition of different ages and brands by electronic nose. Cluster analysis method is Group Average Method, and standardizes the data before the cluster analysis [22]. (each data point minus its average point in the sensor column then divided by the standard deviation in its sensor column)

Fig. 6 is the cluster result of Group A, the different age of the wine under the same brand, clustering of brandies of different brands was conducted according to the age of wine, firstly brandy of similar age can be divided into the same class, and then samples of different aging stage can be classified. The similar test result can be seen compared with the principal component analysis (Fig. 2) when the average distance of the classification is 5.0, three different types of the wine are less than 20 years, 20-30 year and $30-46$ years.

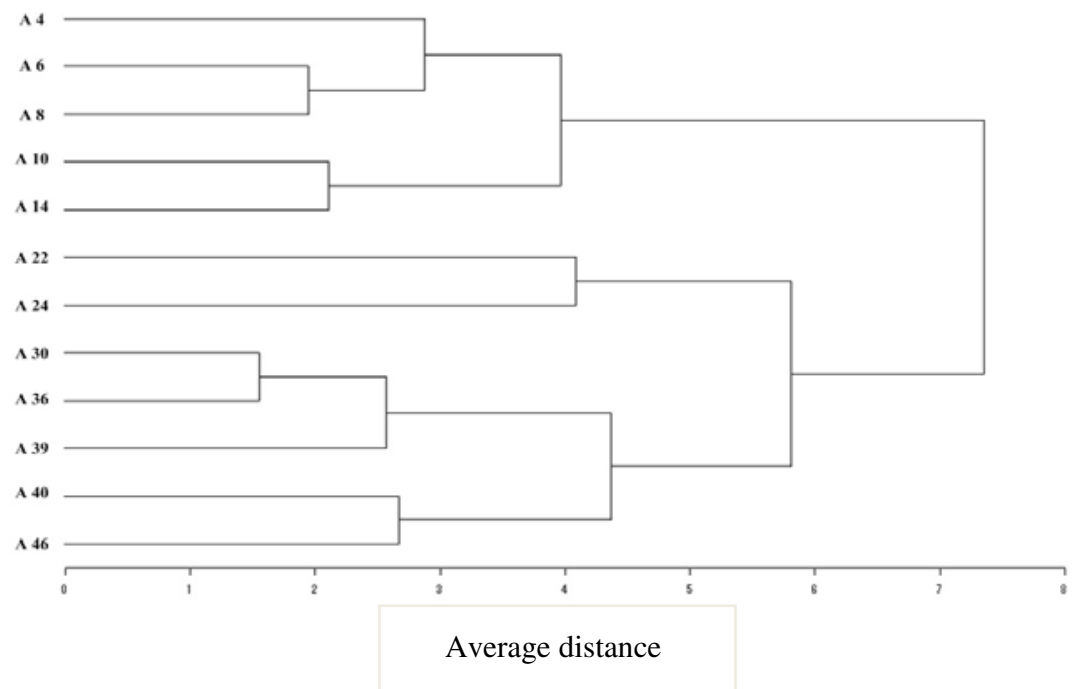

Fig. 6. Tree diagram from clustering analysis in Group Average method for Group A

Cluster analysis results also showed in a regular pattern after cluster analyzing from the Group A, B and C. As we can see in the Fig. 7, three different brands and ages of the brandies were mixed together, clustering firstly process in the same brand of similar age. When the average distance is 3.89 , the cluster can be divided into four groups: Group A, 4-14 years, Group B, 2-6 years, Group B, 7-15 year and Group C, 1-5 years, the brand element is now in the dominant place which illustrated that aroma 
components have significant differences when the age of the wine is under 20 years, electronic nose can distinguish the three brands. When the average distance is 5.43 , the cluster can be assorted into three categories: Group A, B and C wine age less than 20 years, Group A, 22-24 years, Group A, 30-46 year, the component of the wine age placed the most important role during this time, the information reflected from electronic nose to different brand of the wine has been reduced.

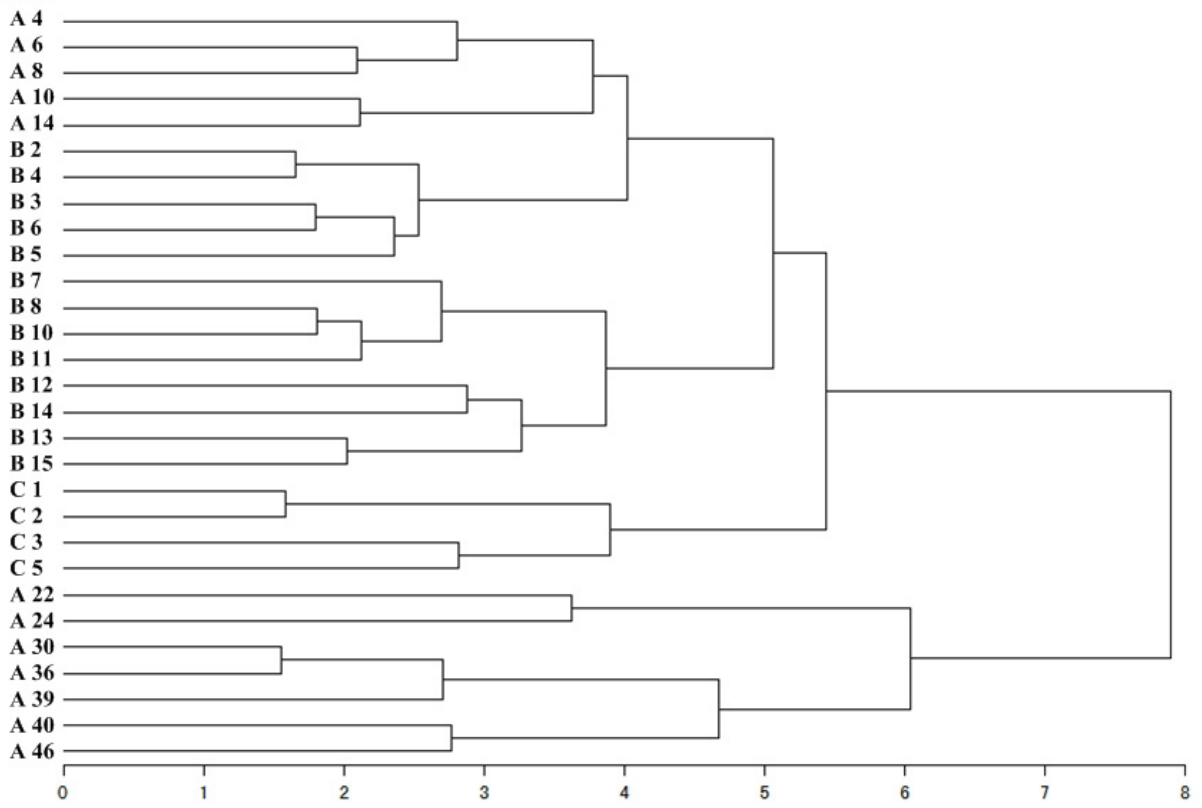

Average distance

Fig. 7. Tree diagram from clustering analysis in Group Average method for Group A, B and C

\section{Conclusions}

GC-flash Electronic nose was used in present study to analyze 29 kinds of raw brandy with different ageing years. It illustrated the identification effect of electronic nose on raw brandy ages and brands, using principal component analysis and clustering analysis.

The principal component of wine sample A, B, C was analyzed. The principal component analysis represents the basic information of original data, and it also presents a good separation of brandy with different ages. The first principal component can reflect the wine age information. Principal component analysis also indicated that stage differences exist among the different brandy ages. Even though the sample age 
difference is big, distance between the brandy samples with small age difference is close; thereby several "ages' stage" was obtained.

Due to the large age difference among three samples, brandy samples with close age differences were chosen to study the identification function of electronic nose among different brands. Although the difference of age exists in different brands, principal component could still be useful in the identification among different brands. In addition, principal component analysis can reflect certain age information. The results indicated that when electronic nose distinguishes the brands of raw brandy, they can accept some wine age differences.

The clustering analysis was conducted on Group A, B and C. It was a process of clustering wine according they ages, which aggregated the brandy with close ages. Clustering analysis was performed on three sample groups together. Clustering process presented same brand wine with close ages firstly. As for the samples with age less than 20 years, clustering according to different brands, brands differences were obvious. When the age gap was big (more than 20 years), brands identification was not good, but age difference became obvious. This result could be caused by the characteristics of brandy themselves. This phenomenon will make contribution to the use of electronic noses on brandy brands and ages identification. When a model was established, brandy ages should be taken into account. When electronic noses can identify wine age and wine brands correctly, the age difference between different brands could be allowed. However, model establishment should minimize the age differences, building models according to different brands wine with different ages respectively.

\section{References}

1. Jiang, Z., Li, J.: Study on relation between components and ages of brandy. J. Food and Fermentation Ind. 34(3), 137-140 (2008) (in Chinese)

2. Schaller, E., Bosset, J.O., Escher, F.: 'Electronic noses' and their application to food. J. LWT - Food Sci. Technol. Int. 31(4), 305-316 (1998)

3. Pinheiro, C., Rodrigues, C.M., Schfer, T., Crespoet, J.: Monitoring the aroma production during wine-must fermentation with an electronic nose. J. Biotechnol. Bioeng. 77(6), 632-640 (2002)

4. Pearce, T.C., Gardner, J.W., Friel, S., Bartlett, P.N., Blair, N.: Electronic nose for monitoring the flavor of beers. J. Analyst. 4(118), 371-377 (1993)

5. Kojima, H., Araki, S., Kaneda, H.: Application of a new electronic nose with fingerprint mass spectrometry to brewing. J. J. Am. Soc. Brew. Chem. 63(4), 151-157 (2005)

6. Lozano, J., Arroyo, T., Santos, J.P., Cabellos, J.M., Horrillo, M.C.: Electronic nose for wine ageing detection. J. Sens. Actuators, B 133(1), 180-186 (2008)

7. Marti, M.P., Busto, O., Guasch, J.: Application of a headspace mass spectrometry system to the differentiation and classification of wines according to their origin, variety and ageing. $\mathrm{J}$. Chromatogr. A 1057(1-2), 211-217 (2004)

8. Garc, A.M., Aleixandre, M., Gutierrez, J., Horrillo, M.C.: Electronic nose for wine discrimination. J. Sens. Actuators, B 113(2), 911-916 (2006)

9. Pornpanomchai, C., Suthamsmai, N.: Beer classification by electronic nose. In: International Conference on Wavelet Analysis and Pattern Recognition, pp. 333-338. IEEE Press, Hong Kong (2008) 
10. Lozano, J., Santos, J.P., Sayago, I., Gutierrez, J., Horrillo, M.C.: Identification of typical wine aromas by means of an electronic nose. J. IEEE Sens. 6(1), 173-178 (2006)

11. Cynkar, W., Dambergs, R., Smith, P., Cozzolino, D.: Classification of Tempranillo wines according to geographic origin: Combination of mass spectrometry based electronic nose and chemometrics. J. Anal. Chim. Acta 660(1-2), 227-231 (2010)

12. Aleixandre, M., Gonzalez, J.A., Sayago, I., Ferrnandez, M.J., Gutierrez, J., Horrillo, M.C.: Analysis of grape variety and denomination of origin of several wines with an artificial nose. In: Electron Devices, pp. 309-311. IEEE Press, Santiago de Compostela (2009)

13. Berna, A.Z., Trowel, 1.S., Clifford, D., Cynkar, W., Cozzolino, D.: Geographical origin of Sauvignon Blanc wines predicted by mass spectrometry and metal oxide based electronic nose. J. Anal. Chim. Acta 648(2), 146-152 (2009)

14. Arroyo, T., Lozano, J., Cabellos, J.M.: Evaluation of wine aromatic compounds by a sensory human panel and an electronic nose. J. Agric. Food. Chem. 57(24), 11543-11549 (2009)

15. Ragazzo-Sanchez, J.A., Chalier, P., Chevalier, D., Calderon-Santoyo, M., Chommidh, C.: Identification of different alcoholic beverages by electronic nose coupled to GC. J. Sens. Actuators, B 134(1), 43-48 (2008)

16. Ragazzo-Sanchez, J.A., Chalier, P., Chevalier, D., Calderon-Santoyo, M., Chommidh, C.: Off-flavors detection in alcoholic beverages by electronic nose coupled to GC. J. Sens. Actuators, B 140(1), 29-34 (2009)

17. Guadarrama, A., Iniguez, M., Souto, J., de Saja, J.A., Feranadez, J.A.: Array of conducting polymer sensors for the characterisation of wines. J. Anal. Chim. Acta 411(1-2), 193-200 (2000)

18. Berna, A.Z., Trowell, S., Cynkar, W., Cozzolino, D.: Comparison of metal oxide-based electronic nose and mass spectrometry-based electronic nose for the prediction of red wine spoilage. J. Agric. Food. Chem. 56(9), 3238-3244 (2008)

19. Gao, Y., Liu, Y., Li, Y., Li, J., Shi, B., Ni, Y.: Electronic nose fingerprint of liquor of main flavor types. J. Liquor-Making Sci. Tech. 5, 38-41 (2008) (in Chinese)

20. Liu, H., Niu, Z.: Comparison of total volatile basic nitrogen detection models in fishmeal based in electronic nose. J. Chin. Soc. Agric. Eng. 26(4), 322-326 (2010) (in Chinese)

21. Liu, M., Oan, L., Tu, G., Liu, P.: Determination of egg freshness during shelf life with electronic nose. J. Chin. Soc. Agric. Eng. 26(4), 317-321 (2010) (in Chinese)

22. Aleixandre, M., Lozano, J., Gutierrez, J., Sayago, I., Fernandez, M.J., Horrillo, M.C.: Portable e-nose to classify different kinds of wine. J. Sens. Actuators, B 131(1), 71-76 (2008) 